\title{
THE POLITICS OF PERFORMATIVITY IN TRANSCOMMUNICATION AND ITS COMMUNICATIVE/EXPRESSIVE FITNESS: TOWARDS A GENERAL OUTLINE
}

\author{
STANISŁAW PUPPEL
}

\section{Introduction}

Embodied agents (EA) inhabit the Earth. As physical entities they are organized into species inhabiting the Earth in a great magnitude and variety (see e.g. Mora et al., 2011). In the present paper a number of assumptions are made, namely that: (a) EAs' being in the World (Aristotle's ousia) involves the most essential dynamic characteristic of 'performativity'. The later may be defined as the acting/expressive potential of any embodied agent, (b) humans as a species are a concretization of the EAs who form a global pool of embodied agents inhabiting the Earth, (c) all EAs are involved in doing 'politics' which, in the Aristotelian sense, is the art of craftsmanship carried out by a universal highly symbiotic and synergistic hylomorphic design. The latter comprises the following: material causes (or simply 'matter'), formal causes (or simply 'structure'), efficient causes (or simply 'functions'), and final cause of acting for any embodied agent. Doing politics consists in the accomplishment by the hylomorphic design of the ever embracing political imprint on all EAs by means of the following dyad of 'political' consequences: strong presence and long duration (longevity) of any EA.

Following Aristotle, it is further assumed that the hylomorphic design outlined above is a reconciliation of both nature (teleological phusis) and laws, principles and customs (nomos), all tightly integrated in polis which, according to Aristotle, is an 
embracing and collaborative compound whole rather than a mere aggregation of constituents. Subsequently, doing politics by the EAs in the sense outlined above necessarily involves the phenomenon and efficacy of 'performativity' or the ability of an embodied agent to act appropriately in time and space (cf. Dourish, 2001).

All human embodied agents who, like all the embodied agents on Earth, are viewed as expressive (i.e. acting/performing) agents, are further assumed to be able to transcommunicate. The latter property (with an emphasis on 'trans') involves an ability to communicate between/among all the individual human agents across the vast expanse of the linguistic resources, institutions, cultures, ethnicities, languages, and communication orders. That is why in the paper, all the human embodied agents are referred to as the 'transcommunicators'. As such, in order to be communicatively effective, successful, and comfortable, they must demonstrate an appropriate degree of 'fitness' (or 'adaptive' power, see e.g. Wright, 1932; Provine, 1986) which may be defined as resulting from man's ontological-epistemological (i.e. semiotic, see e.g. Nöth, 1990) grounding on Earth as the primary carrier, coupled with man's environmental sensibility and subsequent operational integrity such that in any behavioural act a goal (or a set of goals) may be easily accomplished.

As a result of the above considerations, a Performativity Principle (hence PP) may be put forth. It may be tentatively formulated in the following way:

PPa: performativity is a pervasive phenomenon in the sense that all the embodied agents possess and demonstrate performativity (see e.g. McKenzie, 2001),

$\mathrm{PPb}$ : embodied agents communicate/express and by doing so they perform,

PPc: therefore, all the embodied agents (including the human transcommunicators) perform by communicating/expressing and communicate/express by performing.

\section{The Universal Performance Machine}

According to PP, the human EAs as transcommunicators are conceived of as performing and communicating at the same time. It is further assumed that they are doing so while being constrained by an integrated collaborative/interactive hylomorphic design which may be referred to as the Universal Performance Machine (hence UPM). In the perspective outlined in the present account, the UPM is a three-layer EA sustainability design which is composed of the following sub-layers representing three distinct though closely integrated layers (levels) of EA overall communicative/expressive fitness:

I. The Grand Design of Life (GDL)

II. The Imperial Tetragon of Embodiment (ITE)

III. The Lord of the Rings (LR). 
A graphic representation of the UPM is shown below:

The Universal Performance Machine (UPM)

(a hylomorphic compound/design)

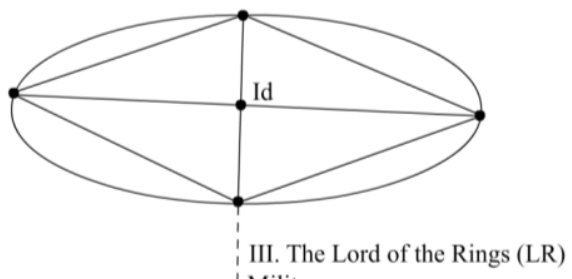

The resource-dependent ultimate exo-level (strong HCA-relative level)

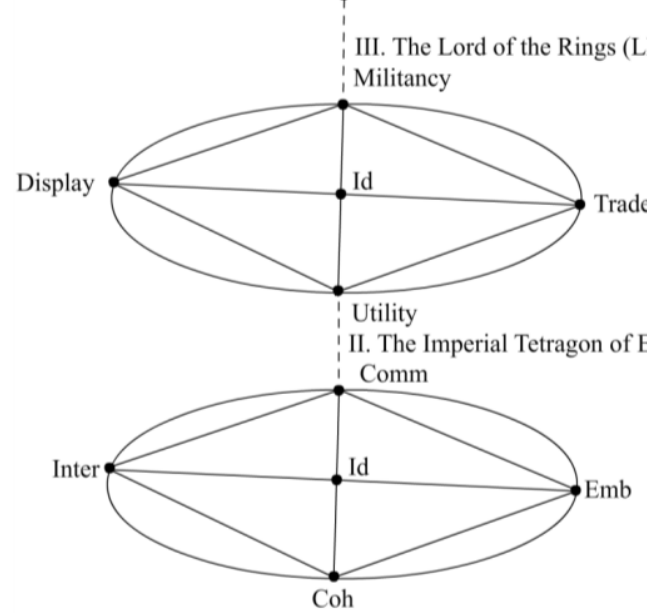

The embodiment-dependent extended meso-level (weak HCA-relative level)

The biology-dependent primary endo-level (HCA-neutral level)

I. The Grand Design of Life (GDL)

Fig. nr 1. The Universal Performance Machine

The three levels will be briefly described below.

\subsection{The Grand Design of Life (GDL)}

The GDL constitutes the lowest, down to Earth, layer of communicative/expressive fitness. It is the biology-dependent primary endo-level on which all the embodied agents (including the human agents as the communicating agents, hence referred to as HCA) experience the primary biological performativity which is stretched, as it were, between the following four nodes: Embodiment (i.e. where all the agents obtain their embodied/essential/material status, best expressed by the following ontological statement: "Here I am"), Cohabitation (i.e. where all the embodied agents are properly aligned against each other, best expressed by the ontological statement: "I am not alone"), Interactivity (i.e. where all the embodied agents interact with each other thus turning action into meaningful interactive inter-species and intra-species behaviours, best expressed by the ontological statement: "I bump/run into others"), 
and Communication (i.e. where all the embodied agents share their resources in acts of communicative behaviours, best expressed by the ontological statement: "I share my resources with others"). The GDL is represented schematically as follows:

\section{The Grand Design of Life (GDL)}

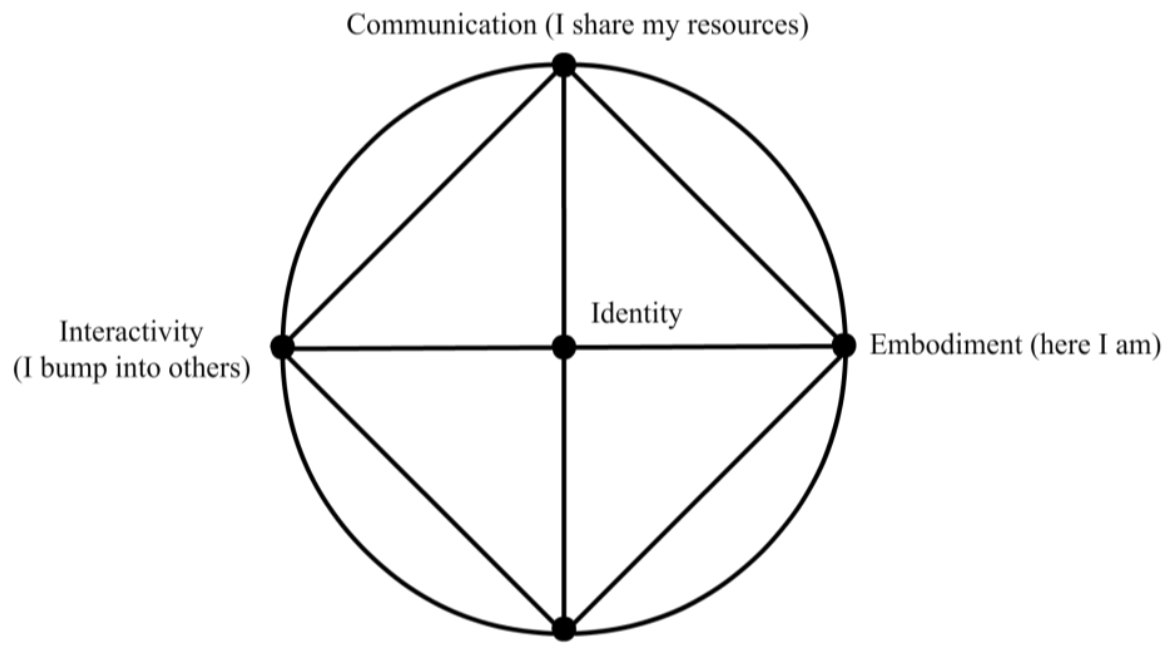

Cohabitation (I am not alone)

The primary biological level of performativity:

the biology dependent parameters can be organized into a stable matrix:

$$
\left[\begin{array}{c}
\text { Embodiment } \\
\text { Cohabitation } \\
\text { Interactivity } \\
\text { Communicaton }
\end{array}\right]
$$

The politics of separation and binding of the embodied agents

Fig. nr 2. The Grand Design of Life

As can be noticed. the GDL as the primary biological (i.e. biology-dependent) level represents pre-expressive performativity which can be organized into a stable matrix consisting of the four parameters of embodiment, cohabitation, interactivity, and communication by means of which the embodied agents perform the basic politics of 'strong presence' which is realized by means of both embodied agent separation and embodied agent binding, coupled with the equally significant political consequence of 'long duration'. Both determine the 'material identity' of the embodied agents on the endo-level of performativity. 


\subsection{The Imperial Tetragon of Embodiment (ITE)}

The ITE constitutes the middle (meso-) layer of the embodied agent communicative/expressive fitness. It is the embodiment-dependent and extended meso-level (cf. Puppel, 2009) on which all the embodied agents experience the extended performativity which is assumed to be stretched between the following four nodes (where the nodes compete for the highest, imperial/dominant, values under appropriate conditions): Militancy (i.e. where all the embodied agents conform to some kind of 'militancy ecology' while always demonstrating a certain degree of aggressiveness/combativeness directed outwards, e.g. towards other embodied agents), Trade-offs (i.e. where all the embodied agents conform to a 'trade-off ecology' by

\section{The Imperial Tetragon of Embodiment (ITE)}

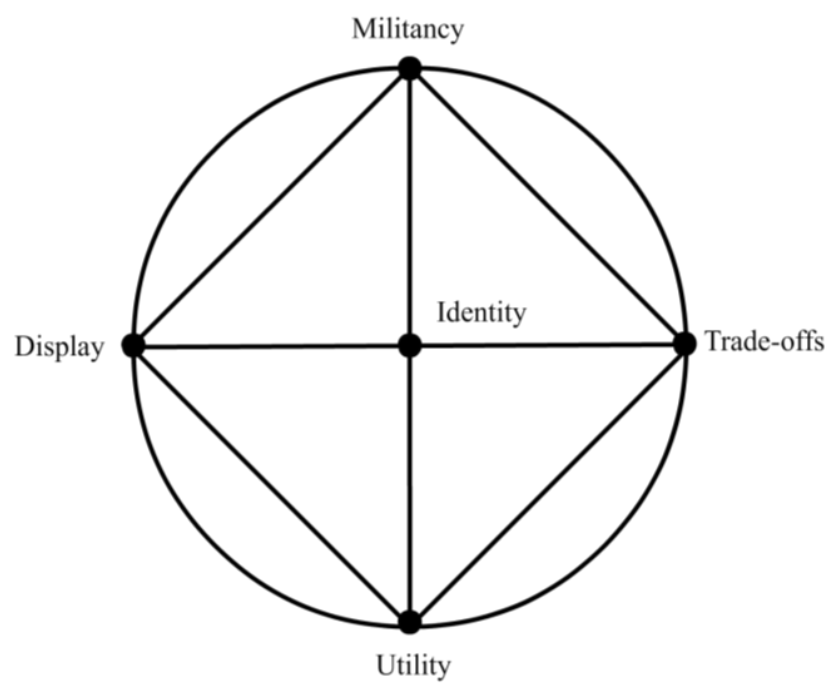

The extended embodiment-dependent meso-level of performativity: the embodiment-dependent parameters can be organized into a highly changeable matrix:

$$
\left[\begin{array}{c}
+/- \text { Militancy } \\
+ \text { /-Trade-offs } \\
+/- \text { Utility } \\
\text { +/-Display }
\end{array}\right]
$$

The politics of context-sensitive embodiment (embodied agents)

Fig. nr 3. The Imperial Tetragon of Embodiment 
means of demonstrating a certain degree of trade-offs, or operational routine compromises, while interacting with other embodied agents), Utility (i.e. where all the embodied agents conform to a 'trade-off ecology' by means of demonstrating a certain degree of trade-offs, or operational routine compromises, while interacting with other embodied agents), Utility (i.e. where all the embodied agents conform to some kind of 'utility ecology' by means of demonstrating a certain degree of concern for economic advantages (or benefits) connected with the embodied agent's possession of any resources and their possible (including altruistic) exchanges with other embodied agents), and Display (i.e. where all the embodied agents are necessarily immersed in 'display ecology' by means of demonstrating their overall display identity, such as the pervasive visage attributes in the form of their beneficial attractiveness, cf. Krebs and Davies, 1997). The ITE is represented schematically as follows.

As can be noticed, the ITE as an extended meso-expressive level of performativity can thus be organized into the following four-parameter matrix (where all the parameters are not only highly context-sensitive but also ontogenetically changeable and are therefore highly changeable in their potentially imperial/dominant appearances): militancy, trade-offs, utility, display. It follows from the above that it is by means of the activation of the ITE matrix and interplay of its constituent highly context-sensitive parameters that the politics of context-sensitive embodied agent mesoperformativity is accomplished.

\subsection{The Lord of the Rings (LR)}

The LR, placed on top of Figure nr 1, constitutes the ultimate language and nonlanguage resource-dependent exo-layer of human embodied agent communicative/expressive fitness. It is the most elaborated culture-determined level on which all the human embodied agents experience and practice the most extended and most sophisticated dynamics of performativity connected with inter-transcommunicator and intra-transcommunicator communicative behaviours by means language resource management. In the perspective applied in the present model, communication is stretched between the following four 'rings' which determine the transcommunicators' (also referred to as the 'human communicating agents', hence HCA) 'communicative competence': the language resource ring, the navigable spaces ring, the communicative styles ring, and the communicative niches ring. In addition, all transcommunicator communicative practices and behaviours are immersed, as it were, in the ever-embracing proclivity of 'affect' (see e.g. Gregg and Seigworth, 2010). The LR is represented schematically as follows: 


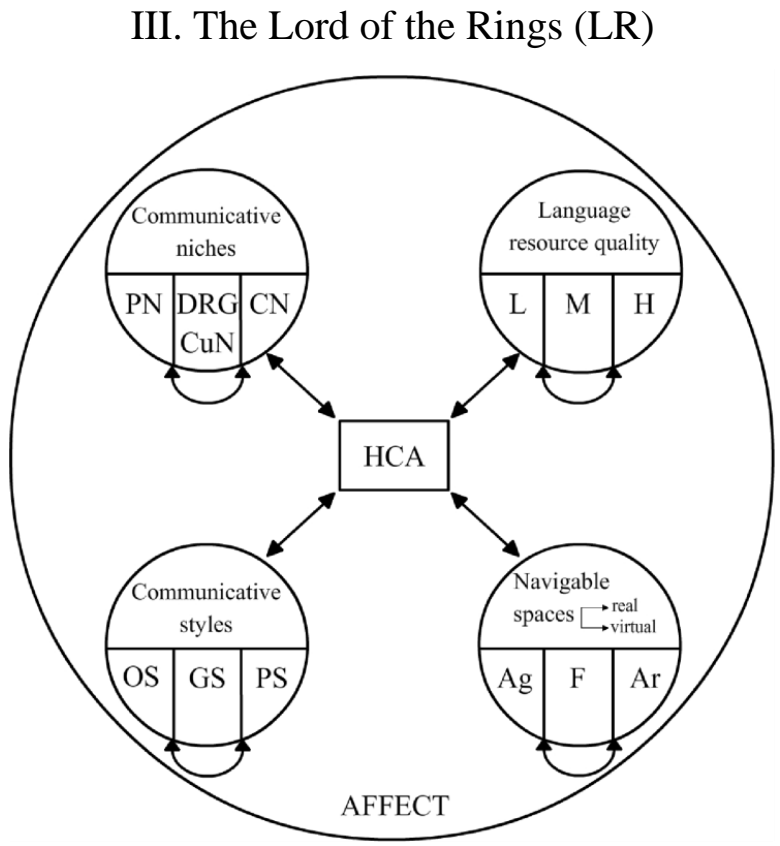

The ultimate resource-dependent exo-level of performativity:

the resource-dependent parameters can be organized into highly changeable matrices with context- and resource-dependent parameters which are subject to affect as an ever-present and ever-encompassing indicator of all the levels.

The politics of context-sensitive resource management by the HCA (quality and quantity)

Fig. nr 4. The Lord of the Rings

The rings are briefly characterized below:

\section{(a) The language resource quality ring}

In the model developed in the paper, the language resources which every individual transcommunicator (or human communicating agent, HCA) has a chance to develop in his/her individual ontogenetic history and may thus have access to may be defined as low, medium, and high, respectively. Obviously, the medium quality of the resources is the one which clearly separates the low and the high resources. This is indicated by the semicircular bidirectional arrow placed underneath the ring.

\section{(b) The navigable spaces ring}

Transcommunicators are assumed to manage the resources in two types of navigable spaces: 'real' (physical) space of transcommunicator contact and 'virtual' space of contact. In addition, the types of contact take place in three major HCA 
contact spaces, the space of the 'Agora', the most widely available communicative space, where HCA contacts are highly haphazard, unplanned and uncommissioned, the space of the 'Forum', a narrowly available communicative space, where HCA contacts are highly systematized, planned and commissioned, and the space of the 'Arena', also widely available communicative space, but where the HCA contacts are additionally impregnated with $\mathrm{HCA}$ attempts at winning the contacts with other HCAs. In this ring, the 'Forum' space seems to reside in the middle of the ring which is indicated by the semicircular bidirectional arrow placed underneath the ring.

\section{(c) The communicative styles ring}

With respect to the communicative styles ring, the HCAs may be characterized as belonging to basically three types of transcommunicators: the 'Oskar Syndrome' (OS) HCA (named after Günter Grass' novel The tin drum, where the main literary figure, Oskar Matzerath, has been depicted by the author as being able to drum vigorously but in a highly limited, repetitive and thus highly predictable manner), the 'Gulliver Syndrome' (GS) (named after Jonathan Swift's masterpiece Gulliver's travels, where the main figure, Gulliver, visits the land of the tiny people (the Lilliputians of Lilliput) and of the giants (in Brobdingnag), and the 'Petronius Syndrome' (PS) (named after Henryk Sienkiewicz's Nobel Prize-winning novel Quo vadis, where one of the main characters, Petronius, has been depicted by the author as arbiter elengantiarum, that is, a master of sophistication and unchangeable and unperturbed elegance).

The three Syndromes are assumed to signify three different communicative styles which the HCAs are capable of applying in changeable communicative contexts and variable communicative encounters or three different styles which they have been able to develop in their individual ontogenetic histories along with the development of language resources. Thus, the 'Oskar Syndrome' represents lowquality resource transcommunicators and therefore basically low quality performativity and expressiveness (via verbal and non-verbal performances), the 'Gulliver Syndrome' represents high-quality resource and highly adjustable transcommunicators (i.e. those who represent adjustable performativity and who are able to adjust their communicative behaviours appropriately depending on the context of the communicative encounters). Finally, the 'Petronius Syndrome' is assumed to represent high-quality resource transcommunicators who are not willing to adjust the use of their resources according to the dictates of the context (i.e. whose performativity is rigidly set on the use of the maximally high resources). In this ring, the 'Gulliver Syndrome' is a syndrome mediating between the Oskar Syndrome and Petronius Syndrome which is properly emphasized by the semicircular arrow placed underneath the ring. 


\section{(d) The communicative niches ring}

As regards the communicative niches ring, every HCA may be characterized as being able to realize the linguistic-communicative potentials of the following three niches: the 'daily routine and general culture niche' (DRGCuN) in which most (if not all) of the transcommunicators 'reside', as it were, and where most daily communications take place by means of medium and low quality language resources, the 'professional niche' (PN) in which the transcommunicators are obliged to use high language resources in professional communicative encounters, and the 'citizenship niche' $(\mathrm{CN})$ in which the transcommunicators are confronted with the highly specialized legal registers of language resources (e.g. constitutions, codes, conventions, charters, treaties, declarations, covenants, warranties, etc.) developed in the course of our social life. In this ring, the DRGCuN is the niche mediating between the $\mathrm{PN}$ and the $\mathrm{CN}$ which is properly indicated by the semicircular bidirectional arrow placed underneath the ring. The four rings are therefore rendered collectively as the 'linguistic-communicative complex' (LCC).

\section{Some conclusions}

The present discussion has prompted the following conclusions:

(a) Performativity is pervasive and is based on prior embodiment which is understood as embodied agent presence and embodied agent separation.

(b) The hylomorphic design presented above generates the overall planetary identity of all the embodied agents.

(c) The EAs demonstrate the politics of performativity which rests on the synergistic activities of the Universal Performance Machine based on the Performativity Principle.

(d) The particular variety of EA, the human transcommunicator (or the human communicating agent), depends in his/her communicative behaviour on the total synergy-induced interplay of the entire LCC, that is, of the four rings of: language resource quality, navigable spaces, communicative styles, and communicative niches.

In other words, the HCAs depend in their EA performances on synergy-induced LCC (hence SILCC). Or, they all move along the SILCC ROAD where strong HCA dependence on the proclivities of the hylomorphic design make them more or less resource- and context-conscious performers.

\section{References}

Barker, E. 1995. Aristotle "Politics". Oxford: Oxford University Press.

Dourish, P. 2001. Where the action is: the foundations of embodied interaction. Cambridge, Mass.: The MIT Press. 
Gregg, M. and G.J. Seigworth, (eds.). 2010. The affect theory reader. Durham, NC: Duke University Press.

Krebs, J.R. and N.B. Davies, (eds.). 1997. Behavioural ecology: an evolutionary approach. $4^{\text {th }}$ ed. Oxford: Blackwell.

McKenzie, J. 2001. Perform or else: from discipline to performance. London: Routledge.

Mora, C., Tittensor, D.P., Adl, S., Simpson, A.G.B. and B. Worm. 2011. "How many species are there on earth and in the ocean?". PLoS Biology 9.8. e1001127 DOI: 10.1371/journal. pbio.1001127, 2011.

Nöth, W. 1990. Handbook of semiotics. Bloomington, IN: Indiana University Press.

Provine, W.B. 1986. Sewall Wright and evolutionary biology. Chicago: The University of Chicago Press.

Puppel, S 2009. "Remarks on the sustainability of natural languages in the cultural-institutional perspective". In: Puppel, S. and M. Bogusławska-Tafelska, (eds.). New pathways in linguistics. Olsztyn: University of Warmia and Mazury, Institute of Modern Languages and Literature. 275-286.

Wright, S. 1932. "The roles of mutation, inbreeding, crossbreeding, and selection in evolution". Proceedings of the Sixth International Congress on Genetics 1. 356-366. 